\title{
Ce que la perspective de l'apprentissage et de l'enseignement contextualisés authentiques peut apporter pour optimaliser la qualité pédagogique des stages d'externat
}

\section{What can bring the authentic situated learning and teaching theoretical framework to optimize the education quality of clinical clerkships?}

\section{Dominique VANPEE ${ }^{1}$, Mariane FRENAY ${ }^{2}$, Véronique GodiN ${ }^{1}$ et Denis BÉDARD ${ }^{3}$}

1 Université catholique de Louvain, Belgique, Faculté de Médecine, cellule de développement pédagogique (CDP) du secteur santé

2 Chaire UNESCO de pédagogie universitaire, Université catholique de Louvain, Belgique

3 Centre d'études et de recherche en enseignement supérieur (CERES), Faculté d'éducation, Université de Sherbrooke, Québec, Canada

Manuscrit reçu le 7 juin 2007 ; commentaires éditoriaux formulés aux auteurs le 30 juillet 2008 ; accepté pour publication le 6 février 2010

Mots clés :

Cognition contextualisée ou située ;

compagnonnage cognitif
Résumé - Problématique : La qualité pédagogique des stages d'externat représente un véritable défi pour toute faculté de médecine. Cette période spécifique de formation représente en effet un moment crucial dans la formation des étudiants en médecine pour consolider, progressivement, les compétences professionnelles du médecin. Cette qualité est pourtant régulièrement remise en question. Messages clés : Nous proposons une lecture de la qualité pédagogique des stages à la lumière du cadre théorique de l'apprentissage et l'enseignement contextualisés authentique (AECA) comme défini récemment par Frenay et Bédard. Ce cadre théorique propose deux grands principes pédagogiques qui sont d'une part l'authenticité du contexte et d'autre part le compagnonnage cognitif. Chacun de ces deux grands principes est composé de sept conditions d'action guidant la planification et la réalisation d'activités d'apprentissage et d'enseignement tenant particulièrement compte des contextes professionnels où seront transférées les connaissances et les compétences acquises. Ce cadre théorique nous apporte ainsi un éclairage non seulement sur la définition des contextes d'apprentissage propices au transfert, mais aussi sur la qualité de l'accompagnement des étudiants au plan du traitement de l'information qu'ils sont appelés à réaliser au moment d'utiliser leurs connaissances. Conclusions : Nous argumentons à démonstration que le cadre théorique de l'AECA décrit récemment dans la littérature de pédagogie générale peut être transposé au contexte des stages cliniques d'externat. 


\section{Keywords:}

Situated cognition; cognitive apprenticeship
Abstract - Problematic: The educational quality of clinical clerkships represents a real challenge for all medical faculties. This specific training period is indeed crucial for medical students to consolidate progressively their professional competencies. The quality of clerkship training is regularly being discussed. Key messages: We propose the analysis of the educational quality of clerkships in light of the theoretical framework of Authentic Situated Learning and Teaching (ASLT) recently described by Frenay and Bédard. This theoretical framework proposes two main teaching principles, namely the context authenticity and the cognitive companionship. Each principle is composed of seven conditions of action in order to guide the planning and the realization of training and teaching activities, taking specifically into account the professional context in which these acquired competencies and knowledge will be transferred. This theoretical framework enlightens us not only on the definition of training contexts favourable to transfer, but also on the quality of the tutoring process in terms of students' capacity to use their knowledge when treating information. Conclusions: This study demonstrates that the ASLT theoretical framework, recently described in general medical education literature, can be transposed to the context of clinical clerkships.

\section{Introduction}

La qualité pédagogique des stages constitue un véritable défi pour toute faculté de médecine. Cette période spécifique de formation représente en effet un moment crucial dans la formation des étudiants en médecine pour développer et consolider, progressivement, les compétences professionnelles du médecin.

L'une des responsabilités premières des facultés de médecine est de former des médecins compétents et réflexifs. Pour ce faire, quel que soit le moment du cursus envisagé (pré clinique ou clinique), le défi pédagogique est de penser et planifier l'articulation des apprentissages concernant à la fois les connaissances déclaratives et les connaissances d'action. Le dispositif de l'apprentissage par problèmes a été développé pour apporter une solution à ce problème durant le cursus préclinique. Nous voulons proposer ici l'idée que, dans le cadre de la partie clinique du cursus, une planification des activités de stage s'inspirant de la perspective de l'apprentissage et l'enseignement contextualisés authentiques (AECA) permettrait d'apporter une solution homologue.

L'objectif de ce travail est ainsi de discuter l'intérêt potentiel du cadre conceptuel de l'AECA, tel qu'il a été défini par Frenay et Bédard, au regard de la problématique des stages ${ }^{[1,2]}$. Nos propos seront délibérément limités aux stages d'externat durant la partie clinique de la formation initiale pré-graduée, bien que certaines analyses puissent être transposées à d'autres milieux de formation.

\section{Contexte et problématique}

Notre question de départ est la suivante : les stages d'externat sont-ils généralement conçus et pédagogiquement exploités comme des endroits propices au développement de compétences cliniques ?

Le concept de compétence est défini de manière variée selon les auteurs mais nous reprendrons ici la définition proposée récemment par Jacques Tardif : "une compétence est définie comme un savoir agir complexe qui prend appui sur la mobilisation et la combinaison efficaces d'une variété de ressources internes et externes à l'intérieur d'une famille de situations ${ }^{[3]}$. En pratique, on considère qu'une personne est compétente lorsque, confrontée à une situation inédite mais comportant un certain nombre de caractéristiques communes à d'autres situations de la même famille, elle est capable de mobiliser un certain nombre de ressources qui, combinées entre elles, lui permettront de résoudre la situation. Cette notion de ressources est fondamentale pour la compétence puisque ce sont ces ressources qui sont 
mobilisées et intégrées pour résoudre la situationproblème.

La pratique du médecin clinicien répond le plus souvent au type de processus suivant : après l'anamnèse et l'examen clinique (analyse de la situation) d'un patient qui présente un problème de santé (le nouveau problème à résoudre auquel il est confronté), le médecin doit être capable de sélectionner et d'activer les ressources pertinentes (par exemple, mobiliser ses connaissances, mettre en œuvre des habiletés de communication, etc.) et de les utiliser (en les intégrant et en les combinant) pour apporter une réponse satisfaisante à la situation de son patient.

Les ressources mobilisables face à une situation-problème sont de deux types : les ressources internes et externes. Sans entrer dans les détails, on peut dire que les ressources internes sont celles qui appartiennent à un individu donné, c'est-à-dire : toutes les connaissances qu'il possède (connaissances déclaratives et connaissances d'action), les habiletés qu'il maîtrise, les attitudes qu'il a intériorisées, etc. Les ressources externes sont, quant à elles, celles qui peuvent être mobilisées en dehors de la personne. Ce sont les ressources matérielles (le formulaire des médicaments, le livre de référence, les revues, les examens complémentaires adéquats, ...), les ressources socio-relationnelles (le collègue à qui on demande un avis, ... ) et les ressources procédurales (l'utilisation des recommandations clinique ou guidelines, ....).

L'éclairage de la psychologie cognitive ${ }^{[4]}$ permet de comprendre pourquoi la formation théorique conduit les étudiants à développer surtout un bagage de connaissances déclaratives, peu ou pas mobilisables en situation de résolution d'un problème comme la prise en charge d'un patient; en effet, la transformation de connaissances déclaratives en connaissances d'action constitue, en tant que telle, un nouvel apprentissage. L'une des problématiques pédagogiques principales de nos cursus de formation est ainsi, certainement, le découplage entre la théorie et la pratique. Selon notre expérience, les stages sont trop souvent conçus comme des moments d'application de connaissances enseignées et apprises ailleurs et trop rarement comme des moments et des contextes exploités pour susciter et orienter les apprentissages, grâce aux activités d'enseignement et d'évaluation. Ce découplage théorie-pratique explique vraisemblablement l'observation fréquemment rapportée par les enseignants-cliniciens : les étudiants éprouvent des difficultés à appliquer les notions théoriques apprises à partir des cours à la prise en charge d'un patient particulier. Nous citerons un simple exemple tiré d'une situation vécue en salle d'urgence : pour un patient en crise d'épilepsie (secondaire à une hypoglycémie), aucun des quatre étudiants n'avait pensé à demander un dosage de la glycémie... Ils avaient pourtant tous appris, dans le cadre de leurs cours théoriques de diabétologie et de neurologie, que «l'hypoglycémie peut provoquer de multiples problèmes neurologiques dont la crise d'épilepsie ». Cependant, ils n'avaient pas été en mesure de mobiliser, face à ce patient et à ce moment-là, certaines des ressources dont ils disposaient pourtant déjà. Un tel constat ne doit pas nécessairement conduire à une remise en cause radicale des cours magistraux mais vise à en signaler les limites concernant les apprentissages qu'ils soutiennent lorsque ces activités sont complètement décontextualisées et qu'elles ne se préoccupent pas explicitement de la construction de connaissances transférables et contextualisées.

Les étudiants, en stage comme ailleurs, construisent leurs connaissances à partir de ce qu'ils savent déjà. Ces connaissances antérieures sont essentielles car ce sont elles qui permettent à l'étudiant d'établir des liens avec les nouvelles informations qui lui sont fournies, afin de les traiter de manière signifiante. Pour que cela soit possible, il est cependant indispensable que ces connaissances soient mobilisables pendant l'action, non inertes, ce qui exige notamment qu'elles soient fortement organisées en mémoire à long terme, comme l'ont notamment démontré les travaux de Bordage ${ }^{[5]}$. Lors du stage, l'étudiant va aussi acquérir de nouvelles connaissances, qu'il devra 
intégrer aux connaissances déjà présentes dans sa mémoire à long terme, créer ainsi un réseau de connaissances fonctionnellement réutilisables. Il est ainsi très important que les formateurs et les maîtres de stage réfléchissent à la manière de favoriser la création de tels liens en contexte de stage, de manière à ce que les connaissances résultant des apprentissages effectués, grâce à des stratégies cognitives et métacognitives guidant leur application, soient intégrées, plus facilement transférables, ce qui implique qu'elles combinent à la fois des connaissances déclaratives, procédurales conditionnelles.

Ces conditions sont-elles souvent réunies dans le cadre des stages proposés aux externes ? Pour répondre à cette question, il est utile d'évaluer si les stages favorisent l'indexation des nouvelles connaissances construites en mémoire à long terme autour de situations, de contextes et de tâches professionnelles complètes, à l'occasion d'activités de résolution de problèmes mal définis et complexes. À l'instar des ouvrages de référence qui sont indexés en bibliothèque (ce qui facilite leur recherche et l'accès aux connaissances qu'ils renferment), les connaissances en mémoire (souvent organisées en mémoire à long terme sous la forme de «blocs de connaissances »-chunk of knowledge) ont avantage à être indexées (rattachées) à des structures cognitives comme des scripts pathologiques ou des scripts d'action (connaissances procédurales). Ce faisant, il sera plus aisé (et possible) pour le médecin qui traite un patient de mobiliser les «blocs de connaissances » pertinents en lien avec les symptômes observés et le diagnostic à poser. À défaut, c'est exiger que la mémoire de travail du médecin mobilise de façon quasi exhaustive (et à plat) toutes les connaissances qui pourraient être pertinentes par rapport au problème clinique, ce qui crée les conditions d'une surcharge cognitive préjudiciable.

Dans la littérature médicale, la qualité pédagogique des stages est malheureusement trop souvent mise en défaut et ce, à des degrés divers ${ }^{[6]}$. Les conclusions du travail de Daelmans sont claires à ce sujet : «we conclude that conditions for adequate workplace learning are poorly met and that clerkship experience show huge inter-student variation » (nous concluons que les conditions favorables à un apprentissage en situation de stage ne sont pas réunies de façon satisfaisante et que les expériences de stages proposées aux étudiants présentes d'énormes variations - traduction libre) ${ }^{[7]}$. Remmen a aussi montré que les stages cliniques ne fournissent pas automatiquement un environnement d'apprentissage adéquat pour les étudiants en médecine parce que les ressources d'apprentissage ne sont pas utilisées de manière optimale, que la majorité de l'enseignement et de l'encadrement y est faite par des jeunes médecins en formation, sans expérience d'encadrement, et que les expériences des étudiants restent trop «passives », compte tenu de la nature de cet encadrement ${ }^{[8]}$. Comme le dit Chamberland, «les activités proposées aux étudiants lors des stages doivent être en accord avec les acquis des sciences de l'apprentissage en général, c'est-à-dire permettre aux étudiants d'être actifs, de construire eux-mêmes leurs connaissances et stratégies à partir de leur bagage antérieur, et ce, dans un contexte signifiant et motivant, qui présente des tâches authentiques et qui favorise le rappel, l'élaboration et le transfert des connaissances » ${ }^{[9]}$. Nous pensons comme beaucoup «qu'il ne suffit pas d'exposer un étudiant à des patients et à des problèmes de santé mais qu'il est nécessaire que ces situations soient systématiquement exploitées pour induire et orienter des apprentissages significatifs et que, dans l'optique d'une professionnalisation, ces problèmes soient abordés dans leur réelle globalité et complexité » ${ }^{[10]}$.

Pour nous, la qualité pédagogique d'un stage résulte non seulement de l'exposition clinique (les opportunités d'apprentissage) mais aussi de la qualité de l'encadrement qui sera fourni à l'étudiant pour que ces opportunités d'apprentissage puissent être le point de départ du développement de réelles compétences cliniques.

Notre expérience sur le terrain montre que deux idées préconçues sont fréquemment rencontrées chez les enseignants cliniciens :

- une première a trait à la conviction illustrée par des 
propos tels que : «ceux qui viennent chez moi apprennent beaucoup en m'observant travailler». De tels propos font référence à un « compagnonnage simple », qui comporte peu ou pas d'interactions cognitives entre le professeur et l'étudiant. Si ce type de compagnonnage est intéressant, il n'est probablement pas suffisant. En effet, la simple observation n'offre pas autant de support, ni ne favorise autant d'apprentissages qu'un accompagnement qui met explicitement l'accent sur les dimensions cognitives. En effet, si le niveau minimal du compagnonnage tient au fait même qu'un expert se place en position de support ou d'appui par rapport à un novice quant à l'apprentissage d'habiletés ou de compétences, fonction essentiellement dédiée à des apprentissages de nature comportementale, un compagnonnage cognitif (cognitive apprenticeship) implique un rôle additionnel de support, centré sur l'explicitation des aspects cognitifs qui soutiennent l'action (connaissances, stratégies, ....).

- Une deuxième idée préconçue peut être illustrée par des propos tels que : «le stage dans mon service offre à l'étudiant l'opportunité de rencontrer beaucoup de pathologies différentes..., l'étudiant observera beaucoup de choses utiles à son développement clinique ». Une telle conception est issue de la perspective de la psychologie humaniste qui postule que l'étudiant en stage sait ce qui est essentiel pour sa propre évolution et son épanouissement personnels. L'environnement riche et varié dans lequel est placé l'étudiant est censé lui permettre d'opérer les choix nécessaires à son développement, l'étudiant devant choisir lui-même les activités qu'il a le goût de réaliser.

L'enseignant qui s'inspire des principes de la psychologie cognitive et qui connaît les avantages d'un environnement riche, conçoit aussi qu'il peut intervenir directement pour soutenir l'étudiant dans la sélection des éléments les plus utiles à son apprentissage. L'enseignant peut dès lors intervenir pour aider le stagiaire à se définir des buts réalistes, à développer le contrôle personnel de ses réussites et de ses échecs, selon une progression vraisemblable, dans une marche vers une autonomie de plus en plus grande.

\section{Un cadre conceptuel pertinent : la perspective de l'apprentissage et de l'enseignement contextualisés authentique}

Les stages sont certainement des endroits privilégiés pour développer les compétences cliniques. Le cadre conceptuel de (AECA) tel qu'il a été défini récemment par Frenay et Bédard ${ }^{[1,2]}$ apporte un éclairage potentiellement intéressant par rapport aux problématiques pédagogiques des stages.

Ce cadre théorique propose deux grands principes pédagogiques qui sont d'une part, l'authenticité du contexte et, d'autre part, le compagnonnage cognitif. Chacun de ces deux grands principes est composé de sept conditions d'action, guidant la planification et la réalisation d'activités d'apprentissage et d'enseignement et tenant particulièrement compte des contextes professionnels dans lesquels les connaissances et les compétences acquises seront transférées. Ce cadre théorique apporte ainsi un éclairage non seulement sur la définition des contextes d'apprentissage propices au transfert, mais aussi sur la qualité de l'accompagnement des étudiants au plan du traitement de l'information qu'ils sont appelés à réaliser au moment d'utiliser leurs connaissances.

La perspective socio-cognitiviste est à la base de ce cadre conceptuel, la majorité des auteurs sur lesquels s'appuie le cadre de l'AECA étant en effet dans cette perspective. Parmi les plus significatifs citons Lave et Wenger pour la perspective sociale et la notion de contextualisation ${ }^{[11]}$; en lien avec ce dernier élément se situent notamment Greeno ${ }^{[12,13]}$, Brown et al. ${ }^{[14]}$, Collins et al. ${ }^{[15]}$, mais aussi Harley ${ }^{[16]}$. Des liens sont aussi faits avec le concept d'apprentissage expérientiel. L'approche expérientielle met l'accent sur le processus d'apprentissage, ses effets chez la personne et le sens de sa compétence $^{[17]}$. Mandeville, se référant aux travaux de Courtois et Pineau ${ }^{[18]}$, précise que «l'approche expérientielle implique un contact direct - avec soi, les autres, l'environnement - et réfléchi. L'expérience n'est pas automatiquement formatrice » ${ }^{[17]}$. C'est 
Tableau I. Conditions associées au principe de l'authenticité du contexte.

\begin{tabular}{|l|}
\hline Principe de l'authenticité du contexte \\
\hline 1. respect du contexte de mobilisation \\
\hline 2. développement de compétences \\
\hline 3. situations complètes et complexes \\
\hline 4. contenus pluridisciplinaires \\
\hline 5. situations-problèmes multidimensionnelles \\
\hline 6. situations-problèmes diversifiées \\
\hline 7. solutions, conclusions, interprétations multiples \\
\hline
\end{tabular}

également ce que propose l'AECA. Enfin, la « réflexion sur l'action », deuxième condition du principe de compagnonnage cognitif, s'inscrit directement dans les travaux de Schön ${ }^{[19]}$.

\section{Premier principe : I'authenticité du contexte (Tableau I)}

Ce premier principe insiste sur le fait que les démarches d'apprentissage et d'enseignement ancrent les connaissances dans un contexte qui doit être le plus proche possible de la réalité - on parle de contexte authentique -, si l'on souhaite favoriser le transfert des apprentissages. Il importe ici de bien évaluer quelle est la réalité professionnelle à laquelle les compétences visées permettent d'accéder pour pouvoir juger adéquatement de l'authenticité des situations d'apprentissages proposées. Pour l'étudiant en stage, c'est à partir de ces contextes que les gestes posés prendront un sens véritable.

Le concept général de cognition contextualisée ou située (situated cognition) est tiré des travaux de Brown, Collins et Duguid et fait appel à deux idées majeures ${ }^{[14]}: 1$ ) le traitement de l'information est indissociable du contexte dans lequel il prend place et 2) le contexte d'apprentissage et d'enseignement influence de manière déterminante la représentation et l'utilisation qui seront faites des connaissances. Toute connaissance semble donc ainsi contextualisée. «Elle est à la fois le produit de l'activité réalisée, du contexte «socio-relationnel » l'entourant et de la culture de laquelle elle provient ou dans laquelle elle est utilisée. ». ${ }^{[1]}$
Sept conditions peuvent soutenir des dispositifs pédagogiques qui visent à respecter ce principe d'authenticité du contexte.

\section{Condition 1 : respecter le contexte de mobilisation (ou de transfert) des apprentissages visés}

C'est la première condition à prendre en compte pour favoriser l'authenticité des situations présentées dans un contexte de formation. Les étudiants doivent s'engager dans des situations d'apprentissage qui soient les plus proches possibles de leur futur métier, afin de pouvoir utiliser les connaissances acquises.

On postule ici que, si l'étudiant estime une situation d'apprentissage proche de son futur métier (contextualisation), il sera suffisamment motivé pour s'investir dans la tâche et ainsi apprendre. Un contreexemple est fourni par la réflexion d'un étudiant : « Je viens de finir un stage de transplantation pédiatrique... Je ne vois pas pourquoi il faut mettre des stagiaires dans ce type de service car cela n'a rien à voir avec notre futur métier... Même si l'équipe était sympathique et que je me suis bien plu, je n'y ai pas appris grand-chose... ».

\section{Condition 2 : soutenir le développement de compétences dans les dispositifs d'enseignement-apprentissage}

Cette condition est liée au fait que c'est par l'action (tâches à faire et problèmes à résoudre) que les étudiants sont invités à faire des apprentissages, à maîtriser leurs habilités et à développer progressivement leur autonomie. La compétence ne peut être perçue uniquement comme la réalisation d'une série de «petites tâches successives » car elle est aussi composée des connaissances, des habiletés - telles que la résolution de problème, l'analyse, la communication - et des attitudes appropriées aux domaines de la discipline ${ }^{[20]}$. Dans ce cadre, une plainte très fréquemment émise par les étudiants en stage hospitalier est qu'ils doivent rédiger des dossiers d'admission pour des patients dont le problème de santé 
est déjà identifié. Ils font donc une anamnèse et un examen clinique dans un contexte qui n'est pas celui de la pratique réelle et ils en transcrivent les résultats dans le dossier clinique du patient. Leur raisonnement clinique n'est nullement évalué. Ils assistent ensuite passivement au suivi du patient qui est assuré par les médecins de l'unité d'hospitalisation. Même si un tel constat peut sembler caricatural, il n'est pas exceptionnel que la seule tâche vraiment active pour laquelle on les sollicite soit celle de la récupération des résultats des examens complémentaires et de leur classement dans le dossier, ce qui est objectivement peu propice au développement de compétences utiles à leur futur métier.

\section{Condition 3 : proposer des situations d'apprentissage complètes et complexes}

Pour qu'elles puissent soutenir le développement des compétences des étudiants stagiaires, il est nécessaire que les situations d'apprentissages soient complètes et complexes. Ceci signifie qu'elles doivent intégrer un ensemble de dimensions ou de facteurs entourant la tâche (temps dévolu pour voir le malade, collaboration avec l'équipe d'infirmières, stress de l'urgence,...). Elles doivent aussi, comme dans le milieu professionnel, prendre en compte plusieurs facteurs en interaction les uns avec les autres. Dans ce cadre, il faut éviter de proposer aux étudiants trop de situations d'enseignement et d'apprentissage construites avec des éléments isolés les uns des autres (morcellement) et épurés des conditions réelles. Il convient au contraire, selon cette condition, d'exposer les étudiants le plus tôt possible à des situations complexes, avec une difficulté croissante, tout en veillant à un soutien cognitif adapté (soutien important pour les étudiants les plus novices, que l'on peut estomper au fur et à mesure du développement de leurs apprentissages et de leurs compétences). Elles devront impliquer la mobilisation de plus en plus importante de stratégies ou d'habiletés.

La compétence au raisonnement clinique et le suivi des patients sont des activités complètes et complexes. En effet, plusieurs traits caractérisent un problème complexe (ill-structured problem) : les indices nécessaires à la solution ne sont pas (tous) disponibles d'emblée, la nature du problème évolue au cours de son investigation, la solution n'est pas standardisée mais originale, le problème ne peut pas être résolu avec un haut degré de certitude et les experts sont souvent en désaccord quant à la meilleure solution à mettre en œuvre même lorsque le problème peut être considéré comme résolu ${ }^{[21]}$. Concernant les étudiants en médecine, on leur demande trop souvent de réaliser uniquement le dossier d'entrée du patient. Il s'agit d'une tâche incomplète, d'autant que, comme cela a déjà été mentionné, l'étudiant en unité de soins rencontre essentiellement des patients dont le problème clinique est déjà formulé ${ }^{[22]}$. Comme le dit Chamberland, «l'étudiant en stage est ainsi privé de la collecte et de l'analyse des indices et confiné à des tâches de gestion, d'investigations et de soins. Il en est fréquemment réduit à appliquer a posteriori un processus de raisonnement (stratégie générale) sur un ensemble d'informations (contenu spécifique) qu'il n'aura pas lui-même recueilli, alors même que c'est l'intrication foncière du processus et du contenu qui est la caractéristique fondamentale du raisonnement clinique ${ }^{[23]}$.

\section{Condition 4 : faire référence à plusieurs contenus disciplinaires et favoriser leur articulation}

Pour exercer correctement son rôle professionnel, le médecin fait référence à plusieurs contenus disciplinaires qu'il articule. Il est donc important, durant la formation et en contexte de stage, de laisser une place suffisante à des contenus qui transcendent la «frontière naturelle » de chaque discipline, dans le but de permettre une meilleure intégration des connaissances. Les stages en unités cliniques hyperspécialisées posent problème : si l'étudiant n'a fait qu'un stage en cardiologie, il pourrait croire par la suite que la majorité des dyspnées sont d'origine cardiaque. 


\section{Condition 5 : confronter les étudiants à des situations- problèmes multidimensionnelles de niveau de com- plexité variable}

Ces situations intègrent l'ensemble des dimensions de la profession, non seulement les compétences disciplinaires mais aussi les compétences transversales (habilités à la communication, considérations éthiques, juridiques, socio-économiques,...). En stage, il sera donc primordial d'inviter explicitement l'étudiant à considérer les dimensions autres que celles liés au contenu disciplinaire strictement bio-clinique du champ professionnel dans lequel il se trouve.

\section{Condition 6 : présenter aux étudiants de situations- problèmes diversifiées}

Il est nécessaire de permettre aux étudiants d'apprendre à appliquer leurs connaissances dans des contextes et des situations variées. Les situations présentées devraient ainsi avoir un niveau de diversification et de complexité croissantes au fur et à mesure de leur avancée dans le cursus de formation.

\section{Condition 7 : proposer aux étudiants des solutions, des conclusions ou des interprétations multiples pour une même situation-problème}

Afin de bien respecter le principe de la contextualisation, il convient de proposer aux étudiants des problèmes permettant plusieurs stratégies (efficaces, moins efficaces, voire erronées) et plusieurs solutions. En effet, il est rare qu'en pratique professionnelle, une solution unique soit présente pour une situation-problème donnée.

Cette dernière condition est probablement très importante pour le développement du raisonnement clinique. Durant ce raisonnement clinique, le médecin émet un diagnostic différentiel (interprétation des indices donnés par l'anamnèse et l'examen clinique) pour émettre des conclusions (le(s) diagnostic(s) le(s) plus probable(s)) pour apporter des solutions (le traitement).
Tableau II. Conditions associées au principe de compagnonnage cognitif.

\begin{tabular}{|l|}
\hline Principe du compagnonnage cognitif \\
\hline 1. articulation des connaissances \\
\hline 2. réflexion sur l'action \\
\hline $\begin{array}{l}\text { 3. généralisation et discrimination (des situations } \\
\text { d'apprentissage) }\end{array}$ \\
\hline 4. coaching \\
\hline 5. échafaudage \\
\hline 6. modélisation \\
\hline 7. retrait graduel \\
\hline
\end{tabular}

Second principe : le compagnonnage cognitif (Tableau II)

Le principe du compagnonnage cognitif (cognitive apprenticeship) considère plus spécifiquement la relation pédagogique qui unit l'enseignant aux étudiants, plus particulièrement au plan des processus cognitifs impliqués lors de l'utilisation des connaissances en situation de résolution de casproblèmes ${ }^{[16]}$. Selon ce principe, les différentes formes d'apprentissage et les différentes activités dans lesquelles s'engage un étudiant doivent être «socialement partagées ». En effet, en situation d'apprentissage, l'activité cognitive n'est généralement pas uniquement le lot d'individus isolés mais également le propre d'un groupe d'individus à l'intérieur duquel on retrouve fréquemment un expert. Dans ce cadre, les rôles adoptés par les experts (dans notre cadre, l'enseignant clinicien) sont habituellement variés et adaptés aux situations dans lesquelles ils évoluent. Ces rôles sont implicites pour les experts. Il est ainsi nécessaire que les enseignants cliniciens portent attention à la façon dont les connaissances sont utilisées par l'étudiant. Les stratégies d'apprentissage sont constituées de l'ensemble des activités de traitement de l'information auxquelles se livre l'étudiant pendant l'apprentissage et qui influenceront son processus d'encodage des informations. Pour effectuer des apprentissages à la fois approfondis et signifiants (deep and meaningful learning), plutôt que des apprentissages superficiels et «par cœur» (surface and rote learning), 
il faut que l'étudiant développe toute l'étendue des stratégies cognitives (élaboration, organisation, résolution de problèmes) et métacognitives (évaluation ou jugement porté sur ses activités d'apprentissage et sur son fonctionnement cognitif). Il est généralement admis qu'en suscitant l'engagement des étudiants dans des stratégies d'apprentissage les plus élaborées et les plus exigeantes et en aidant les étudiants à organiser leurs connaissances, on favorise une construction de connaissances moins inertes, plus facilement réutilisables ${ }^{[3]}$.

Le principe du compagnonnage cognitif peut aussi s'énoncer sous la forme de sept conditions (tableau II). Les trois premières font référence directement aux rôles de l'apprenant dans ce processus. Les quatre suivantes font référence aux rôles que peut jouer l'enseignant pour soutenir l'apprentissage et le transfert des étudiants. Les enseignants cliniciens ont ainsi, dans leurs moyens pédagogiques, à activer les conditions propres à l'étudiant-apprenant et à assumer des rôles différents et complémentaires pour soutenir ce processus de compagnonnage cognitif.

\section{Trois conditions liées au rôle de l'étudiant apprenant}

\section{Condition 1 : articuler les connaissances}

L'articulation des connaissances est à la base de tout traitement de l'information ultérieur. Elle permet de comparer et de prendre en compte les connaissances apprises dans différents contextes, ce qui devrait favoriser les processus de transfert. Les connaissances à apprendre doivent être clairement énoncées par l'enseignant et articulées avec des démarches de raisonnement favorisant le traitement de celles-ci. L'étudiant devra donc être fréquemment sollicité par l'enseignant, dans le cadre d'une rétroaction qui dirige l'attention des étudiants vers les compétences et les connaissances qu'ils ont développées ainsi que sur leurs stratégies d'acquisition de ces compétences et de ces connaissances.

\section{Condition 2 : penser de manière réflexive}

Il est nécessaire d'aider les étudiants à développer une pensée critique et progressivement autonome. Pour ce faire, l'étudiant doit être invité à penser de manière réflexive, en portant son attention sur ses actions. Il doit être régulièrement invité ou mis dans la position de poser un regard critique ou évaluatif sur ses actions, par exemple en comparant ses processus de résolution de problèmes avec celui d'une personne experte ou avec celui d'autres étudiants. Pour McLellan, cette réflexion peut avoir lieu dans l'action (experimental thinking, réflexion rapide et immédiate) mais peut aussi prendre place après l'action (reflexive thinking) ${ }^{[24]}$. Une telle démarche, qui vise des habiletés métacognitives, peut donc être conduite soit à titre individuel, soit dans le cadre d'une activité de groupe de pairs supervisée par un tuteur.

\section{Condition 3 : préparer le transfert des apprentissages grâce aux stratégies de discrimination et de généralisation}

Les connaissances à la base du transfert des apprentissages sont une variété particulière des connaissances d'action, dénommées connaissances conditionnelles. Elles sont encodées en mémoire à long terme sous la forme d'une représentation productionnelle de type : plusieurs conditions/une action (si..., si..., si..., alors...) $)^{[4]}$. La construction de ces règles de production nécessite la mise en œuvre des deux processus cognitifs que sont la discrimination et la généralisation, complémentaires l'un de l'autre. La discrimination est l'activité cognitive par laquelle l'apprenant distingue, en identifiant des caractéristiques différentielles, deux entités ou situations, qui peuvent être, en apparence, similaires. La généralisation est l'activité cognitive par laquelle l'apprenant reconnaît que, sous certaines conditions, une action peut s'appliquer à plusieurs cas ou situations, qui peuvent être, en apparence, dissemblables. Pour exercer ces deux processus, il est nécessaire d'alterner des périodes de contextualisation (application des connaissances en lien avec un contexte 
donné), de décontextualisation (théorisation des apprentissages d'où émergent les principes et les modèles) et de recontextualisations (nouvelles situations où seront appliquées les connaissances).

L'apprentissage est un processus fondamentalement individuel mais la motivation à apprendre et le caractère approfondi des apprentissages sont favorisés par l'interaction avec d'autres personnes, pour autant que les tâches d'apprentissage soient suffisamment complexes et qu'elles proposent un défi cognitif.

Pour pouvoir généraliser et discriminer à l'occasion de situations d'apprentissage, il est nécessaire, entre autre, de pouvoir comparer son processus d'analyse et de résolution de problème à celui de ses pairs ou de ses maîtres de stage.

\section{Quatre conditions liées aux rôles de l'enseignant clinicien}

\section{Condition 4 : observer et guider l'étudiant}

Le rôle d'entraînement (coaching) est une condition centrale du compagnonnage cognitif. Il consiste à observer les étudiants durant l'exécution d'une tâche (par exemple lors de la supervision directe d'un étudiant interrogeant et examinant un patient) et à intervenir auprès d'eux lorsque le besoin se fait sentir. Dans ce cadre, le plus souvent, le tuteur prend des notes de ses observations. Il intervient de manière ponctuelle en donnant un commentaire ou un avis aidant l'étudiant à revoir l'état de la situation. Il ne donne pas la solution du cas à résoudre mais aide l'étudiant à reprendre sa réflexion ou à changer de stratégie de résolution de problèmes. Ainsi le tuteur intervient quand l'étudiant a besoin d'une aide particulière pour avancer.

On parle de supervision directe quand le maître de stage observe lui même l'étudiant en train de faire une tâche et indirecte quand l'étudiant, après avoir vu un patient, rapporte les éléments pertinents de l'anamnèse et de l'examen clinique à son maître de stage. En 1993, déjà, Scott montrait que la supervision en milieu clinique était trop souvent laissée au hasard des rencontres et n'était nullement structurée $^{[25]}$. Une publication plus récente relève les mêmes observations : durant l'externat, la supervision est faible et s'effectue le plus souvent sans qu'il y ait eu d'observation directe ${ }^{[6]}$. Dans l'étude de Prince, $31,3 \%$ des étudiants se disent non supervisés, $26,9 \%$ ont un avis mitigé et $41,8 \%$ se considèrent comme correctement supervisés quand ils effectuent un examen clinique ou une anamnèse ${ }^{[26]}$.

Les étudiants disent fréquemment ne pas bénéficier de rétroactions (feedback) durant leur stage. La rétroaction est pourtant être un élément essentiel de l'intervention pédagogique en milieu clinique ${ }^{[27]}$. En effet, la pratique de la médecine est souvent vue comme l'application d'un ensemble de savoirs, d'habiletés et d'attitudes. Pour informer au mieux les étudiants à propos de la qualité de leur performance clinique et les motiver pour qu'ils s'améliorent en permanence, le recours à une rétroaction constructive et spécifique est nécessaire. Dans ce cadre, l'observation directe par un enseignant clinicien d'un étudiant en situation de pratique clinique réelle offre l'opportunité d'apprécier les forces et les faiblesses de l'étudiant et de lui retourner l'information afin qu'il améliore ses performances dans des tâches ultérieures.

La capacité à donner adéquatement de la rétroaction a été identifiée comme étant une des caractéristiques des enseignants-cliniciens experts ${ }^{[28]}$. Sans rétroactions, les étudiants peuvent ne pas percevoir leurs erreurs, construire des conceptions erronées et développer de mauvaises habitudes. Ils peuvent aussi ne pas prendre conscience de la qualité de leur réalisation et abandonner sans le savoir des façons de faire efficaces. Il s'agit là d'un élément fondamental en début de formation clinique, qui fait souvent défaut même s'il ne faut pas occulter le fait que certains auteurs ont identifié que les étudiants avaient souvent du mal à reconnaître qu'une rétroaction leur avait été fournie, surtout si ce moment n'a pas été identifié explicitement par le superviseur ${ }^{[29]}$.

\section{Condition 5 : fournir du soutien à l'étudiant}

Dans une situation d'échafaudage (scaffolding), les étudiants ont à réaliser une tâche complexe et le 
support qui leur est offert varie selon leur degré de maîtrise des compétences. L'enseignant clinicien peut assumer lui-même une partie de la tâche compte tenu de la compétence des étudiants ; il rappelle les informations stratégiques ou même les stratégies pertinentes à considérer dans le cadre de la résolution du problème clinique. Dans la perspective de l'AECA, l'enseignant ne postule pas qu'il sait ce que l'élève devrait savoir mais il accepte que le traitement de l'information qui sera effectué par l'étudiant est particulier et il est soucieux de lui fournir un appui au bon moment afin de favoriser un recours à une démarche structurée et rigoureuse ${ }^{[1]}$.

\section{Condition 6 : modéliser cognitivement la tâche}

La modélisation (modeling) est possible si l'enseignant clinicien rend accessible le traitement de l'information qu'il réalise au moment où il fait l'action. Les étudiants sont placés dans un contexte d'observation où une personne exécute devant eux une tâche professionnelle, selon un protocole de pensée à voix haute. Ainsi, la verbalisation du raisonnement clinique d'un médecin pendant qu'il examine un patient favorise la démarche réflexive de l'étudiant. Les superviseurs aussi peuvent intervenir en agissant comme modèle de rôle explicite à l'égard de la réflexion en action et sur l'action.

\section{Condition 7 : accompagner l'exercice autonome}

La diminution graduelle de l'assistance que l'enseignant apporte à l'étudiant (fading) offre aux étudiants des opportunités d'initiatives et de prise en charge autonome de leur propre démarche de travail. Pour Harley, le niveau de support que devrait offrir l'enseignant est inversement proportionnel à l'expérience des étudiants; au fur et à mesure que leur niveau de compétence augmente, les interventions de l'enseignant devraient diminuer ${ }^{[16]}$.

Le stage devrait idéalement être divisé en trois phases (pas nécessairement toujours linéaires mais avec des retours possibles à une phase précédente suivant l'évolution du stage). La phase d'observation est la période d'appropriation par l'étudiant de son nouvel environnement. C'est aussi un temps d'observation mutuelle du maître de stage et de l'étudiant. À ce stade, le stagiaire porte un regard attentif sur une pratique nouvelle pour lui. La seconde phase est celle de la mise en situation. Si celle-ci se déroule bien, on pourra passer à la troisième phase, celle de l'autonomie (cette autonomie étant bien évidemment relative pour nos étudiants en stage).

\section{Hypothèses de solution et exemples potentiels d'application}

Le stage clinique d'externat constitue théoriquement, au sein de l'ensemble du dispositif de formation initiale pré-gradué, le contexte le plus favorable pour que les étudiants puissent développer leurs compétences médicales. Les étudiants en stage ont la possibilité d'y être confrontés à des problèmes réels complexes et authentiques (opportunités d'apprentissage) et peuvent y être accompagnés dans leur processus de raisonnement (sélection et manipulation des connaissances en fonction d'un problème à résoudre) par un clinicien-enseignant. Pourtant, la qualité pédagogique des stages d'externat est régulièrement remise en question. Encore faut-il, en effet, que ces conditions favorables soient effectivement exploitées dans le cadre d'une stratégie pédagogique. Pour ce faire, il convient en effet que les enseignants cliniciens soient non seulement des experts dans leurs domaines cliniques respectifs mais qu'ils aient aussi suffisamment d'expérience en matière de supervision pédagogique pour appliquer les principes de base tels d'un compagnonnage cognitif de qualité.

Le cadre théorique de l'AECA, qui a été développé en pédagogie générale, nous parait transposable à la problématique pédagogique des stages cliniques et notamment de l'externat. En effet, il fournit des repères pour analyser et apprécier les différentes composantes du stage idéal. Au cours d'un tel stage, il est important d'aider l'étudiant à développer des connaissances fonctionnelles et transférables. Il est 
ainsi primordial de le soumettre à des tâches complètes et complexes, congruentes par rapport à la pratique clinique future, en vue de faciliter les processus de transfert des connaissances. Il est donc illusoire d'espérer que les étudiants développent des compétences de raisonnement clinique si l'on se limite à attendre d'eux qu'ils remplissent, dans un service hospitalier, des dossiers d'admission pour des patients avec un diagnostic déjà connu ou qu'ils observent simplement les médecins seniors du service sans que ceux-ci rendent explicites leur fonctionnement cognitif (compagnonnage simple). Il est par ailleurs important d'exposer les étudiants à des situations d'apprentissage variées, multidimensionnelles et complexes, qui favorisent leur implication cognitive afin de leur permettre graduellement de s'approprier les connaissances utiles et d'interagir efficacement avec la situation.

Le cadre théorique de l'AECA devrait devenir un outil pertinent que ce soit au niveau des formations pédagogiques à mettre en place pour les enseignants-cliniciens mais aussi pour le choix des lieux de stage et pour l'évaluation des lieux de stage par les facultés de médecine. Dans les institutions hospitalières, les enseignants cliniciens n'ont généralement que très peu (voire pas du tout) de formation à l'encadrement clinique. Pour compenser cela, la cellule de développement pédagogique du secteur santé de l'Université catholique de Louvain a prévu de mettre en place des formations spécifiques à l'encadrement clinique. Ces formations seront axées sur les quatorze conditions du cadre théorique de l'AECA.

Le choix des lieux de stage dans la planification d'une formation est un élément crucial. On peut se demander si une formation clinique presqu'exclusivement réalisée dans un milieu hospitalouniversitaire est suffisamment contextualisée par rapport à la pratique d'un futur médecin et si les opportunités d'apprentissage y sont suffisamment représentatives de la pratique ultérieure de la majorité des futurs médecins. Nous pensons que non et qu'une faculté de médecine devrait planifier une partie des stages d'externat dans des hôpitaux généraux, en médecine ambulatoire, en médecine générale et aux urgences.

L'évaluation des endroits de stage est aussi un véritable défi. Nous sommes actuellement en train de terminer la validation d'une grille d'évaluation pédagogique des lieux de stages à partir des différentes conditions du cadre théorique de l'AECA. L'avantage de cette grille est qu'elle devrait permettre d'évaluer nos lieux de stages suivant les principes récents de la recherche en éducation. Pour tout directeur de stage, ce cadre devrait permettre de répondre à une série de questions. Nos stages répondent-ils correctement aux standards pédagogiques actuels en termes de développement de compétence professionnelle? Offrent-ils un environnement propice pour des apprentissages en profondeur? Donnent-ils suffisamment d'opportunités d'apprentissage ? Quelle est la qualité de l'exploitation pédagogique de ces opportunités ? Les étudiants y développent-ils une pratique réflexive ? L'encadrement est-il adéquat? En bref, les stages sont-ils des lieux d'AECA?

\section{Conclusions}

Nous pensons que le cadre théorique de l'AECA, décrit récemment dans la littérature pédagogique générale, peut être transposé au contexte des stages cliniques d'externat. Il est potentiellement intéressant en tant que référentiel pédagogique pour la formation des enseignants cliniciens mais aussi comme outil d'évaluation de la qualité pédagogique des lieux de stages fréquentés par les externes. Toute formation en contexte de stage réunissant les conditions reliées aux deux principes directeurs de l'AECA (l'authenticité du contexte et le compagnonnage cognitif) serait susceptible de favoriser l'intégration des connaissances, le développement des compétences, l'activation et le transfert des connaissances ainsi qu'une perception positive de la tâche de l'étudiant comme apprenant. 


\section{Contributions}

Tous les auteurs ont collaboré de manière étroite à la rédaction de ce travail et aux relectures successives des différentes versions du manuscrit. Dominique Vanpee a transposé dans la pratique des stages le cadre conceptuel de l'apprentissage et de l'enseignement contextualisés authentiques, tel qu'il a été défini par Mariane Frenay et Denis Bédard.

\section{Références}

1. Frenay M, Bédard D. Des dispositifs de formation universitaire s'inscrivant dans la perspective d'un apprentissage et d'un enseignement contextualisé pour favoriser la construction de connaissances et leur transfert. In: A Presseau et M Frenay. Le transfert des apprentissages : comprendre pour mieux intervenir. Québec: Les Presses de l’Université Laval, 2004.

2. Bédard D, Frenay M, Turgeon J, Paquay L. Les fondements de dispositifs pédagogiques visant à favoriser le transfert de connaissances : les perspectives de «l'apprentissage et de l'enseignement contextualisés authentiques ». Res Academica 2000;18:21-47.

3. Tardif J. L'évaluation des compétences (documenter le parcours de développement) Montréal : Chenelière Éducation, 2006.

4. Tardif J. Pour un enseignement stratégique (l'apport de la psychologie cognitive). Montréal : Les Éditions Logiques, 1997.

5. Bordage G, Zacks R. The structure of medical knowledge in the memories of medical students and general practitioners: categories and prototypes. Med Educ 1984;18:406-16.

6. Langevin S, Hivon R. En quoi l'externat ne s'acquitte-til pas adéquatement de son mandat pédagogique ? Une analyse qualitative fondée sur une analyse systématique de la littérature. Pédagogie Médicale 2007;8:7-23.

7. Daelmans HE, Hoogenboom RJ, Donker AJ, Scherpbier AJ, Stehouwer CD, van der Vleuten CP. Effectiveness of clinical rotations as a learning environment for achieving competences. Med Teach 2004;26:305-12.

8. Remmen R, Denekens J, Scherpbier A, Hermann I, van der Vleuten C, Royen PV, Bossaert L. An evaluation study of the didactic quality of clerkships. Med Educ 2000;34:460-4.
9. Chamberland M. Comment exploiter les résultats de la recherche pour améliorer nos activités d'enseignement et d'apprentissage du raisonnement clinique ? Pédagogie Médicale 2005;6:197-199.

10. Jouquan J, Bail Ph. À quoi s'engage t-on en basculant du paradigme d'enseignement vers le paradigme d'apprentissage ? Pédagogie Médicale 2003;4:163-175.

11. Lave J, Wenger E. Situated learning: Legitimate peripheral participation. Cambridge: Cambridge University Press, 1991.

12. Greeno JG. A perspective on thinking. American Psychologist, 1989;44:134-41.

13. Greeno JG. Understanding concepts in activity. In: C Weaver, CR Fletcher et S Mannes (Eds.), Discourse comprehension: Essays in Honor of Walter Kintch. Hillsdale (NJ): Laurence Erbaum, 1994.

14. Brown JS, Collins A, Duguid P. Situated cognition and the culture of learning. Educational Researcher 1989;18:3242.

15. Collins A, Brown JS, Newman SE. Cognitive apprenticeship: Teaching the craft of reading, writing and mathematics. In: LB Resnick (Ed.). Knowing, learning, and instruction: Essays in honors of Robert Glaser. Hillsdale (NJ): Lawrence Erlbaum, 1989.

16. Harley S. Situated learning and classroom instruction. In: H MacLellan (Ed.). Situated learning perspectives. Englewoord Cliffs (NJ): Educational Technology Publications, 1996.

17. Mandeville L. Une expérience d'apprentissage significatif pour l'étudiant. In: D Bédard et J-P Béchard (dir.). Innover dans l'enseignement supérieur. Paris : Presses Universitaires de France, 2009.

18. Courtois B, Pineau G. La formation expérientielle des adultes. Paris : Documentation française, 1991.

19. Schön D. Educating the reflective practitioner, San Francisco: Jossey-Bass, 1987.

20. Hager P, Gonczi A. What is the competence? Med Teach 1996;18:6-19.

21. King PM, Kitchener KS. Developping reflective judgment: Understanding and promoting intellectual growth and critical thinking in adolescents and adults. San Francisco (CA): Jossey-Bass, 1994.

22. Vanpee D, Godin V, Pestiaux D, Gillet JB. Stages dans un service d'urgence : réflexions sur leur apport spécifique dans la formation médicale de base. Pédagogie Médicale 2003;4:89-92.

23. Chamberland M. Les séances d'apprentissage du raisonnement clinique (ARC) : Un exemple d' activité pédagogique contextualisée adaptée aux stages cliniques en médecine. Ann Med Interne (Paris) 1998;49:479-84. 
24. McLellan H. Situated Learning: Multiple Perspectives. In: H. MacLellan (Ed.): situated Learning Perspectives. Englewood Cliffs (NJ): Educational Technology Publications, 1996.

25. Scott, C, Irby D, Gilliland B, Hunt, D. Evaluating clinical skills in an undergraduate medical education curriculum. Teach Learn Med 1993;5:49-53.

26. Prince KJ, Boshuizen HP, van der Vleuten CP, Scherpbier AJ. Students' opinions about their preparation for clinical practice. Med Educ 2005;39:704-12.

27. O'Brien $\mathrm{H}$, Charlin B. Le feedback (ou rétroaction) : un élément essentiel de l'intervention pédagogique en milieu clinique. Pédagogie Médicale 2003;4:184-91.
28. Wright SM, Kern DE, Kolodner K, Howard DM, Brancati FL. Attributes of excellent attending-physician role models. N Engl J Med 1998;339:1986-93.

29. Irby DM. What clinical teachers in medicine need to know. Acad Med 1994;69:333-42.

Correspondance et offprints : Dominique Vanpee. Service des Urgences, Cliniques UCL de Mont-Godinne, 5530 Yvoir, Belgique, 53 avenue Emmanuel Mounier, 1200 Bruxelles, Belgique. Téléphone $+32(0) 81423133$.

Mailto : dominique.vanpee@uclouvain.be 